\title{
METODOLOGÍA PARA LA ESTIMACIÓN DE ÍNDICES DE CAPACIDAD EN PROCESOS PARA DATOS NO NORMALES
}

\section{METHODOLOGY FOR THE ESTIMATION OF CAPABILITY INDICES IN PROCESSES WITH NON NORMAL DATA}

\author{
Erick A. Chacón Montalvan', Vilma S. Romero Romero², \\ Luisa E. Quispe Ortiz ${ }^{3}$, José W. Camero Jiménez ${ }^{4}$
}

\begin{abstract}
RESUMEN
La globalización ha ido intensificando la competencia en muchos mercados. Con el fin de mantener su competitividad, las empresas buscan satisfacer las necesidades de los clientes mediante el cumplimiento de los requerimientos del mercado. En este contexto, los Índices de Capacidad de Proceso (ICP) juegan un rol trascendental en el análisis de capacidad de los procesos. Para el caso de datos no normales existen dos enfoques generales basados en transformaciones (Transformación de Box -Cox y de Johnson) y percentiles (Sistemas de distribuciones de Pearson y de Burr). Sin embargo, estudios anteriores sobre la comparación de tales métodos muestran distintas conclusiones y por ello nace la necesidad de aclarar las diferencias que existen entre estos métodos para poder implementar una correcta estimación de estos índices. En este trabajo, se realiza un estudio de simulación con el objetivo de comparar los métodos mencionados y proponer una metodología adecuada para la estimación del ICP en datos no normales. Además, se concluye que el mejor método a emplear depende del tipo de distribución, el nivel de asimetría de la misma y el valor del ICP.
\end{abstract}

Palabras clave.- Ajuste de distribuciones de frecuencia, Índice de capacidad del proceso, normalidad, Transformación de datos, Simulación.

\begin{abstract}
Globalization has intensified competition in many markets. To remain competitive, the companies look for satisfying the needs of customers by meeting market requirements. In this context, Process Capability Indices (PCI) play a crucial role in assessing the quality of processes. In the case of non-normal data there are two general approaches based on transformations (Box-Cox and Johnson Transformation) and Percentiles (Pearson's and Burr's Distribution Systems). However, previous studies on the comparison of these methods show different conclusions, and thus arises the need to clarify the differences between these methods to implement a proper estimation of these indices. In this paper, a simulation study is made in order to compare the above methods and to propose an appropriate methodology for estimating the PCI in non-normal data. Furthermore, it is concluded that the best method used depends on the type of distribution, the asymmetry level of the distribution and the ICP value.
\end{abstract}

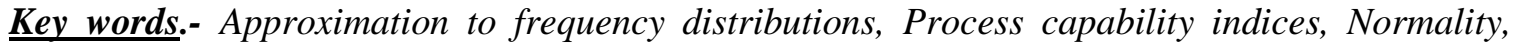
data transformations, Simulation.

\footnotetext{
${ }^{1}$ Egresado de Ingeniería Estadística (FIECS-UNI), Tesista del IRD (Institut de Recherche pour le Développement), ${ }^{2}$ Egresada de Ingeniería Estadística (FIECS-UNI), Tesista del IRD (Institut de Recherche pour le Développement), ${ }^{3}$ Egresada de Ingeniería Estadística (FIECS-UNI), Analista Junior en BCP (Banco de Crédito del Perú), ${ }^{4}$ Catedrático de Ingeniería Estadística (FIECS-UNI), Consultor en Control Estadístico de Procesos.
} 


\section{INTRODUCCIÓN}

La competitividad es un factor primordial que prevalece en el mercado actual de cualquier tipo de producto o servicio. Se ha ido intensificando debido al mundo tan globalizado en el que vivimos, donde la comunicación y la interdependencia de los países conduce a la inevitable unión de culturas y mercados. Por esta razón las empresas hacen mayores esfuerzos por mantener a los clientes en un nivel de satisfacción adecuado. Este nivel de satisfacción dependerá de la capacidad que tenga una empresa para brindar productos que cumplan con las especificaciones del mercado. Por ello, los Índices de Capacidad del Proceso (ICP) son de suma importancia ya que tienen como objetivo cuantificar la calidad del proceso según las especificaciones del mercado. Estas especificaciones están determinadas según un límite de especificación superior (LES) e inferior $(L E I)$. En primer lugar, Juran propuso formalmente el indice Cp en 1974 [1].

$$
C_{p}=\frac{6 \sigma}{6 \sigma}
$$

En donde $(L E S-L E I)$ viene a ser la propagación admitida de las especificaciones y $6 \sigma$ es la propagación actual del proceso, que abarca el 99.73\% de las salidas cuando el proceso está bajo control.

Posteriormente en 1986, Kane discutió el índice $C_{p k}$ como un ICP mejor al $C_{p}[2]$. Con la misma finalidad otros indices fueron definidos como el $C_{p m}$ [3] y el $C_{p m k}$ [4].

Sin embargo, estos ICPS, denominados tradicionales, suponen que la variable de calidad $X$ del proceso subyacente se distribuye normalmente. Supuesto que es vulnerado en distintos procesos de la realidad, por lo que surgió la necesidad de desarrollar ICPS capaces de ser válidos para cualquier tipo de distribución. Ante ello, se puede encontrar dos enfoques generales para afrontar este problema [5].

En primer lugar, mediante la transformación de variables se intenta conseguir datos normales y emplear los ICPS tradicionales; entre las transformaciones más comunes se encuentran las transformaciones de Box-Cox [6] y Johnson [7].

En segundo lugar, se ajustan los datos a una distribución de frecuencia con el fin de calcular percentiles para reemplazar $6 \sigma$ por $\left(P_{99.865}-\right.$ $\left.P_{0.135}\right)$ y la media por la mediana en los ICPS tradicionales. En 1989, Clements propuso utilizar la familia de distribuciones de Pearson [8].

Por otro lado, en el 2005, Liu y Chen propusieron emplear las distribuciones del sistema de Burr XII [9].

Dentro de este contexto se hizo inevitable discutir sobre la relevancia y eficacia de los métodos propuestos. Mediante estudios de simulación comparativos, Ahmad y Abdollahian recomiendan el uso de las distribuciones de Burr XII [10], mientras que Hosseinifard et al proponen el método de transformaciones de potencia como el más adecuado [11].

Por otro lado Czarski, en el 2008, aún recomienda y establece el enfoque de Clements mediante las distribuciones de Pearson como un método óptimo y sin mayores complicaciones para la estimación del ICP [12].

Esta divergencia se debe a que cada trabajo de comparación ha sido elaborado de manera parcial, es decir en un contexto diferente de tal manera que no se ha considerado distintos factores como la distribución bajo estudio, el nivel de sesgo y el valor aproximado del ICP que definen un escenario.

Por lo tanto es necesario brindar una metodología para la estimación del ICP en datos no normales que aclare qué método es adecuado bajo cierto escenario según los factores mencionados.

Por esta razón es razonable realizar un estudio comparativo de simulación que brinde resultados más generales.

Por lo tanto, en el presente trabajo se realiza un estudio de comparación entre los métodos de Clements, Box-Cox, Johnson y Burr con el fin de proponer una metodología que recomiende el uso de cierto método para cada escenario. 


\section{DEFINICIÓN DE ÍNDICES}

\section{Índices de Capacidad para datos Normales}

Existen cuatro tipos básicos de ICP [13], los cuales se muestran en la Tabla 1.

Tabla 1. Tipos de ICP.

\begin{tabular}{|c|c|}
\hline Indice & Características \\
\hline$C P=\frac{\text { LES }-1 F}{60}$ & $\begin{array}{l}\text { Considera } \\
\text { variación del proceso y } \\
\text { no puede detectar la } \\
\text { desviación de la media } \\
\text { del proceso respecto al } \\
\text { centro de las } \\
\text { especificaciones. }\end{array}$ \\
\hline 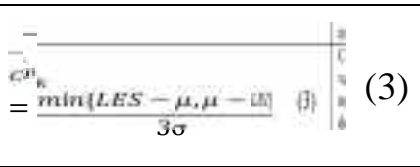 & $\begin{array}{l}\text { Considera la media y } \\
\text { varianza del proceso pero } \\
\text { no puede detectar la } \\
\text { desviación del valor } \\
\text { objetivo. }\end{array}$ \\
\hline$C v n_{1}-\frac{L E S}{e \sqrt{a^{2}+} \frac{E}{(\mu-T)^{2}}}=4$ & $\begin{array}{l}\text { Detecta la desviación del } \\
\text { valor objetivo pero no } \\
\text { puede detectar la } \\
\text { localización de la media } \\
\text { del proceso en el } \\
\text { intervalo (LEI-LES). }\end{array}$ \\
\hline 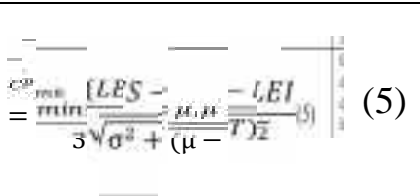 & $\begin{array}{l}\text { Considera la variabilidad } \\
\text { del proceso, desviación } \\
\text { del valor objetivo y la } \\
\text { localización de la media } \\
\text { del proceso en el } \\
\text { intervalo (LEI-LES). }\end{array}$ \\
\hline
\end{tabular}

Donde $\mu$ es la media del proceso, $\sigma$ es la desviación estándar del proceso, $T$ es el valor objetivo y (LES-LEI) son los límites de especificación superior e inferior respectivamente. Además, Juran calificó a los procesos, según el valor del $C_{p}$, de la siguiente manera [1]:

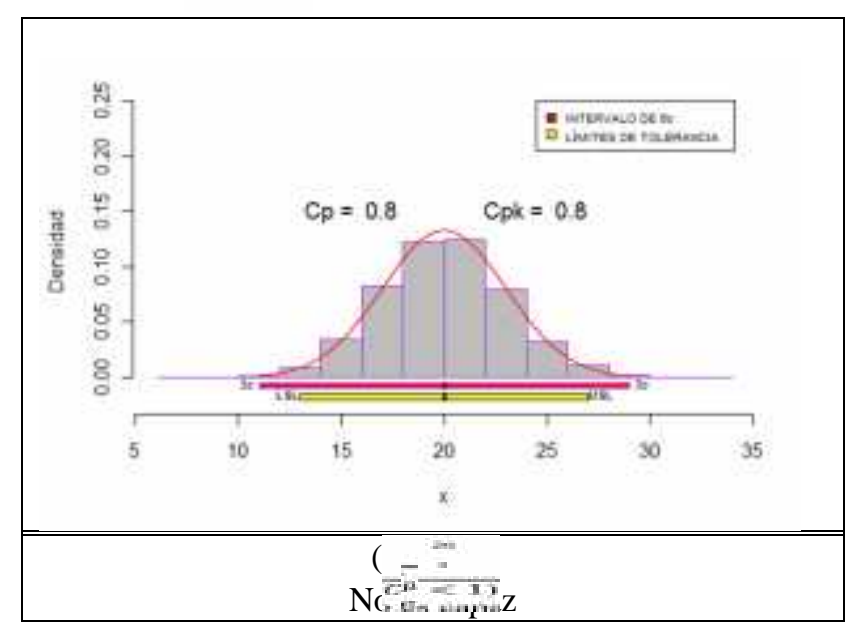

Fig. 1 Proceso no capaz.

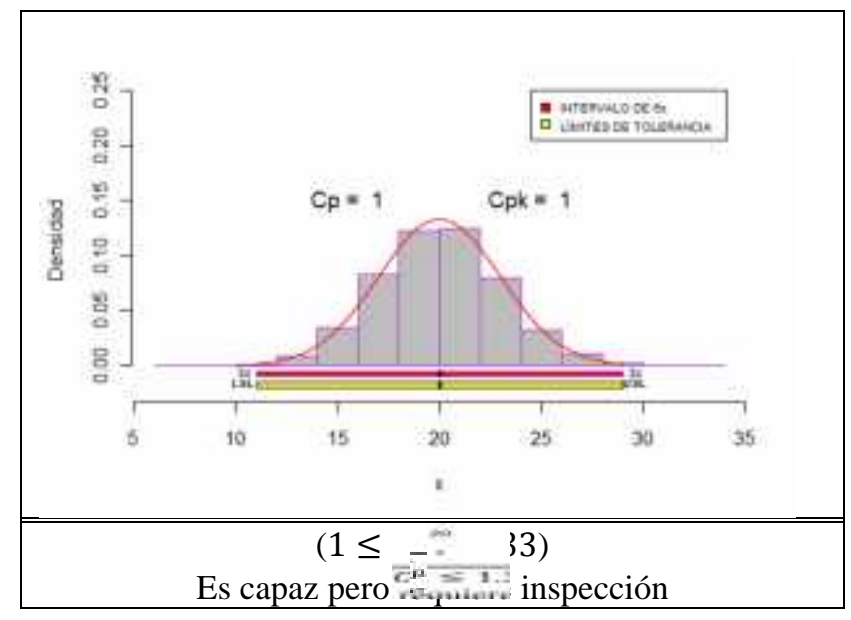

Fig. 2 Proceso capaz con $C p=1$.

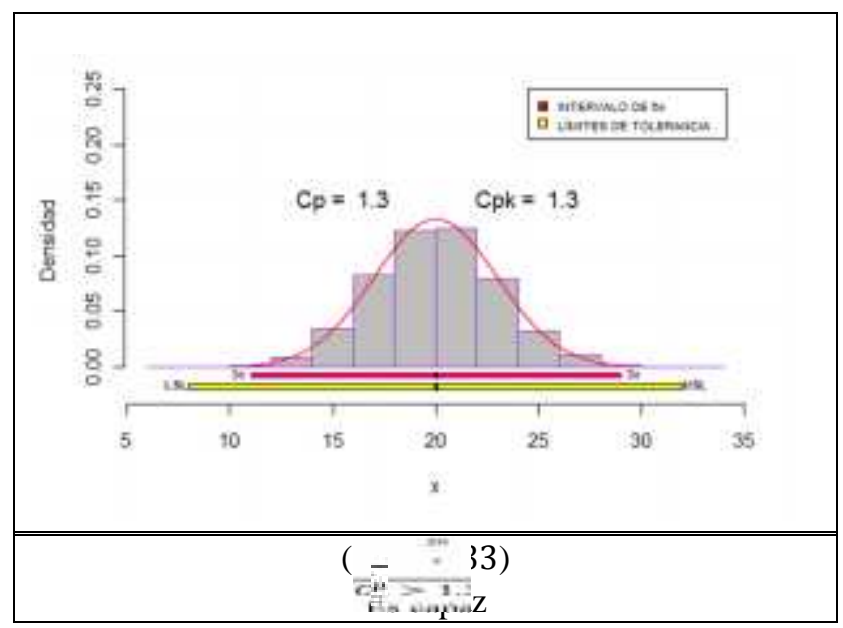

Fig. 3 Proceso capaz con $C p>>1$.

\section{Índices de Capacidad para datos no Normales}

Al tener procesos distribuidos de manera diferente a la distribución normal, se debe considerar a la mediana $(M)$ como medida de tendencia central y la propagación actual del proceso $(6 \sigma)$ debe ser calculada a partir de los percentiles 99.865 y 0.135 de la distribución. Es así, que las fórmulas presentadas anteriormente, quedan definidas de la siguiente manera:

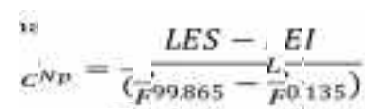

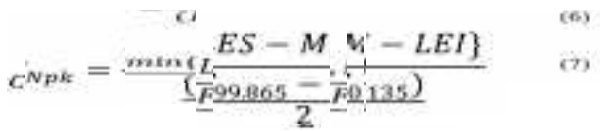




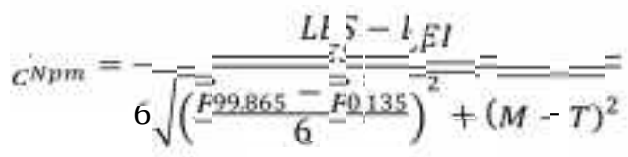

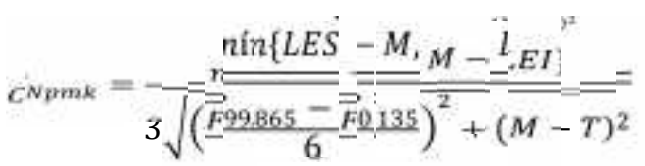

Además, se debe mencionar que las características descritas para los diferentes ICP en datos normales son similares para los ICP definidos para datos no normales. Es decir, cada uno de ellos se diferencia de acuerdo a la capacidad de considerar la variabilidad del proceso, el desvío del valor objetivo y localización de la medida de tendencia central del proceso en el intervalo (LEI-LES).

\section{FORMULACIÓN DEL PROBLEMA DE INVESTIGACIÓN}

Dado que los procesos de la realidad no poseen distribuciones normales, se requiere una adecuada metodología para la estimación de ICP para datos no normales, que permita la integración de diversos factores involucrados como el tamaño de muestra, la distribución que posea el proceso, el nivel de asimetría de la distribución y el valor aproximado del ICP.

\section{IMPORTANCIA Y JUSTIFICACIÓN}

La importancia del estudio radica en la necesidad que tienen las empresas de evaluar de forma adecuada y realista la capacidad de sus procesos, con el objetivo de ofrecer productos acordes a las especificaciones del mercado y lograr que sus clientes tengan un buen nivel de satisfacción.

Además, esta investigación permitirá aclarar algunas discrepancias existentes en distintos estudios comparativos, cada uno con sus respectivas delimitaciones, que se han realizado en los últimos 5 años.

\section{MARCO TEÓRICO}

\section{Métodos de estimación del ICP en datos no normales}

Método de percentiles.- El método de percentiles se basa en los índices definidos en la Tabla 1. Para estimar aquellos índices para datos no normales solo se requicre estimar los percentiles $F_{99.865}$, $F_{0.135}$ y $F_{0.5}$ de modo que puedan ser reemplazados en las ecuaciones de los ICPs para datos no normales (6) - (9). Este método fue propuesto por Clements [8] y es ampliamente usado desde entonces, los métodos más conocidos son los de Clements y Burr.

Método de Clements.- Pearson propuso un sistema para ajustar datos muestrales a curvas de frecuencia en el año 1895 [14]. La ecuación diferencial general para el ajuste a esta familia de distribuciones es de la siguiente manera:

$$
\frac{d y}{d x}=-\frac{y(x+a)}{b 0}+b_{1} x \frac{a}{+b_{2}{ }^{x} \bar{z}}
$$

Donde $a, b_{0}, b_{1}$ y $b_{2}$ son los parámetros de la ecuación. La solución de la ecuación diferencial depende de la naturaleza de lás raíces de la función del denominador $b_{0}+b_{1} x+b_{2} x^{2}$. Para mayor detalle respecto a la solución de

(10) y las distribuciones obtenidas véase [15]. Los valores de los parámetros $a, b_{0}, b_{1}$ y $b_{2}$ son estimados a partir del coeficiente de asimetria y de curtosis.

Con apoyo de la familia de distribuciones de Pearson, Clements fue el primero en proponer las modificaciones en las fórmulas convencionales [8]. Planteó usar esta familia de distribuciones para estimar el valor de los percentiles.

Con los percentiles en mano, se pueden remplazar estos valores en las fórmulas de los ICPs para procesos no normales definidos en (6) - (9).

Método de Burr.- En 1942, Burr encontró un sistema de función de distribución $\mathrm{F}(\mathrm{x})$ que cumplía la generalización de la ecuación de Pearson [16], resultando la siguiente:

$$
\begin{gathered}
f(x \mid c, k)={c k x x_{c-1}\left(d+x_{c}\right)^{-i k+n}} \\
F(x \mid c, k)=1-\left(1+x_{c}\right)^{-k}
\end{gathered}
$$

Con $\mathrm{x} \geq 0, \mathrm{c} \geq 1, \mathrm{k} \geq 1$, siendo $\mathrm{c}$ y $\mathrm{k} \quad$ los parámetros de la distribución y a su vez los coeficientes de asimetría y curtosis respectivamente. 
Burr notó que esta familia de distribuciones tiene una relación especial con los valores de curtosis y asimetría, por lo que sería lógico relacionarlo con los momentos 3 y 4 [17]. Por ello, se relaciona los valores de la desviación estándar, coeficientes de asimetría y curtosis con los parámetros de la Burr XII en las tablas resumen.

Esta distribución captó el interés de muchos investigadores que lo estudiaron a mayor detalle como Takahasi [18], quien señala que la distribución de Burr es una composición de la distribución de Weibull y de Gamma, por lo que se puede introducir una distribución Burr multivariada. Además esta distribución de Burr, conocida en el ámbito económico como SinghMaddala de 2 parámetros [19], es una de las distintas distribuciones llamadas "distribuciones generalizadas log.logisticas".

Con el empleo de este sistema de distribuciones, Liu y Chen proponen, como una variación del método de Clements, usar la distribución Burr XII para una mejor estimación [9].

\section{Transformaciones}

El método de transformaciones se basa en aplicar alguna función $\mathrm{g}(\mathrm{X})$ a la variable de calidad $\mathrm{X}$ de modo que la variable transformada $g(X)$ se distribuya normalmente. Para que los límites sean comparables con la variable transformada se requiere transformar los límites LEI y LES de la forma $\mathrm{g}(\mathrm{LEI})$ y $\mathrm{g}(\mathrm{LES})$ que vendrían a ser los nuevos límites para la variable transformada.

Como ya se tiene la variable y los límites de especificación transformados en las mismas unidades, solo queda estimar la capacidad del proceso mediante los ICPs convencionales. Además, no es necesario volver a la anterior escala ya que los resultados de $g(X)=Y$ son válidos para $\mathrm{X}$, en el caso de los ICP.

Las técnicas de transformación más conocidas y empleadas son la de Box-Cox y la de Johnson, las cuales se presentan a continuación.

Transformación de Box-Cox.- La transformación de Box-Cox fue propuesta por George E.P. Box y David R. Cox. [6]. Esta transformación es usada en variables de respuesta estrictamente positivas, y está dada como sigue:

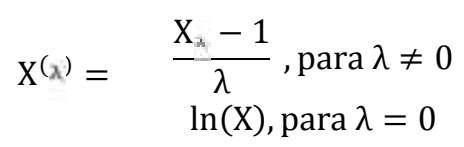

La transformación depende de un solo parámetro $\lambda$, el cual puede estimarse mediante el método de máxima verosimilitud, como fue mostrado por Box y Cox [6].

Luego, el cálculo del Cp se realiza considerando la media y desviación estándar de los datos transformados.

Sistema de Transformaciones de Johnson.Consiste en determinar la distribución, dentro de la familia de distribuciones de Johnson, a la que pertenecen los datos, y realizar una transformación sobre ellos de modo tal que el conjunto de datos se aproxime a una normal [7].

En 1949, Johnson define tres familias de distribuciones para una variable aleatoria $\mathrm{X}$ continua, como sigue:

- SL, que se refiere a $X$ acotada por debajo o Log normal.

- $\mathrm{SU}$ (No acotada), que se refiere a X no acotada.

- SB (Acotada), que se refiere a X acotada.

Se definen cada una de las respectivas transformaciones para este Sistema de Familias de Distribuciones de Johnson, tomadas de Chou et al [7].

- Familia SL de Johnson,

$$
\mathrm{Y}=\gamma+\eta \ln (\mathrm{X}-\varepsilon)
$$

- Familia SU de Johnson,

$$
\mathrm{Y}=\gamma+\eta \sinh ^{-1}\left(\frac{\mathrm{x}-\mathrm{s}}{\mathrm{x}}\right)
$$

- Familia SB de Johnson,

En donde:

$$
Y=\gamma+\eta \ln \left(\frac{x-x-\bar{X})}{(x+\varepsilon-1)}\right.
$$

$\mathrm{Y}$ es la variable transformada, $\mathrm{X}$ es la variable a ser transformada $\mathrm{y}$ se cumple las siguientes restricciones.

$$
\eta, \lambda>0, \quad-\infty<\gamma<\infty, \quad-\infty<\varepsilon<\infty
$$

TECNIA 24 (1) 2014 


\section{METODOLOGÍA}

\section{METODOLOGÍA PARA LA ESTIMACIÓN DEL ICP}

Una metodología adecuada para estimar el ICP en un proceso, normal o no normal, debe considerar distintos factores de acuerdo al escenario en el que se encuentra. Es por ello que la metodología propuesta involucra el tipo de distribución, su nivel de asimetría y el valor aproximado del ICP, con fin de evaluar el escenario y decidir qué método de estimación emplear con el objetivo de minimizar el error. Los factores de entrada definidos son: El tipo de distribución, en nivel de asimetría y el valor del ICP. Adicionalmente como factor intermedio se utilizó el método de estimación del ICP.

\section{METODOLOGÍA DE COMPARACIÓN}

Para la formulación de una metodología para estimar el ICP, en primer lugar debe conocerse bajo qué circunstancias se empleará cada método. Para ello se realiza una comparación entre los métodos de manera experimental mediante un estudio de simulación para valores del ICP iguales a $0.5,1,1.5$ y 2 con el objetivo de caracterizar distintos tipos de procesos respecto a su capacidad, además el tamaño de muestra considerado es 100 para todas las simulaciones.

A diferencia de otros trabajos realizados como el de Hosseinifard et al [11], en donde se hace comparación entre los métodos de transformaciones y los de ajuste de distribución de datos; el de Ahmad y Abdollahian [10], donde se compara los métodos de Clements, Burr y BoxCox, los autores del presente trabajo presentan una comparación más completa.

Por lo tanto, la comparación propuesta debe ser capaz de involucrar distintos factores de modo que no se obtengan conclusiones que solo sean válidas para ciertas situaciones, sino que, por lo contrario, se abarque la mayoría de escenarios posibles modificando los niveles de los factores que se tomen en consideración. Con fines de comparación con otros estudios realizados [10, 11 y 13$]$, se toma como criterio de comparación el $C_{p u}$ y al definir este valor se puede hallar el valor del limite de especificación superior (LES).

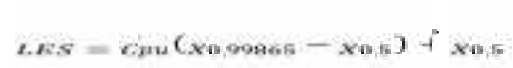

De esta manera el único valor que se tiene que definir es el valor del $C_{p u}$.

\section{Factores a considerar}

Los factores que se consideran son los siguientes:

- El método de estimación; son considerados los métodos siguientes: Box-Cox, Johnson, Método de Clements y el de Burr.

- El tipo de distribución; se considera las distribuciones con mayor importancia en las industrias como es la distribución Gamma, Beta, Weibull y Lognormal. Además, estas distribuciones nos permitirán comparar los resultados con los de [11y 10].

- El nivel de asimetría. Se sabe que la asimetría es el principal motivo de la no normalidad, por eso es considerada. Los niveles de asimetría considerados son: negativo alto, negativo moderado, sin sesgo, positivo moderado $\mathrm{y}$ positivo alto.

\section{Criterio de decisión}

Los métodos aplicados son comparados según el error cuadrático medio ECM.

$$
E C M-E(x-\theta)^{2}=\sum_{i=1}^{n} \frac{\left(x^{i}\right.}{n}-\frac{-\theta)^{2}}{n}
$$

Aquel método que presente menor ECM será considerado como el mejor método estadísticamente.

Los intervalos de confianza no brindan un indicador para determinar cuál de los métodos es mejor pero si son útiles como herramientas visuales para evaluar el comportamiento de los resultados según cada método.

\section{Proceso de simulación}

Los pasos del proceso de simulación son:

Paso 1. Se define un valor objetivo del $C_{p u}=0.5$, 1, 1.5 ó 2. El poder definir el valor del 
parámetro para este tipo de estudios, es de gran ayuda ya que se puede contrastar con el valor estimado que se obtenga de cada método. En [10] se considera los mismos valores para el ICP.

Paso 2. Se define la función de distribución, el nivel de sesgo y sus parámetros.

Paso 3. Con el valor dado del $C_{p u}$ y la función de distribución se calcula el

LES $=\mathrm{C}_{\mathrm{pu}}\left(\mathrm{X}_{0.99865}-\mathrm{X}_{0.5}\right)+\mathrm{X}_{0.5}$.

Paso 4. Se genera una muestra aleatoria de tamaño $\mathrm{n}$ con la función de distribución definida.

Paso 5. Se prueba si la muestra simulada es aleatoria y se encuentra bajo control estadístico mediante la prueba de rachas y el gráfico de control X-R. Si no cumple con los dos requisitos volver al paso 4 .

Paso 6. Se actualiza el número de muestras $m=m+1$ simuladas que se encuentran bajo control y son aleatorias

Paso 7. Se realiza la estimación del $\mathrm{C}_{\mathrm{pu}}$ para la muestra mediante los métodos de Clements, Burr, Johnson y Box-Cox. Cada uno fue definido en la sección II. Y se almacena las estimaciones en una variable RESULTADO.

Paso 8. Si $\mathrm{m}<100$ volver al paso 4 hasta obtener 100 muestras.

Paso 9. Como la variable RESUltAdO consta de 4 columnas y 100 filas que vienen a ser la estimación del $\mathrm{C}_{\mathrm{pu}}$ mediante los cuatro métodos, se calcula el ECM para cada método e intervalos de confianza.

Paso 10. Se evalúa el menor ECM y se decide que el método con aquel valor mínimo del ECM es el mejor estadísticamente.

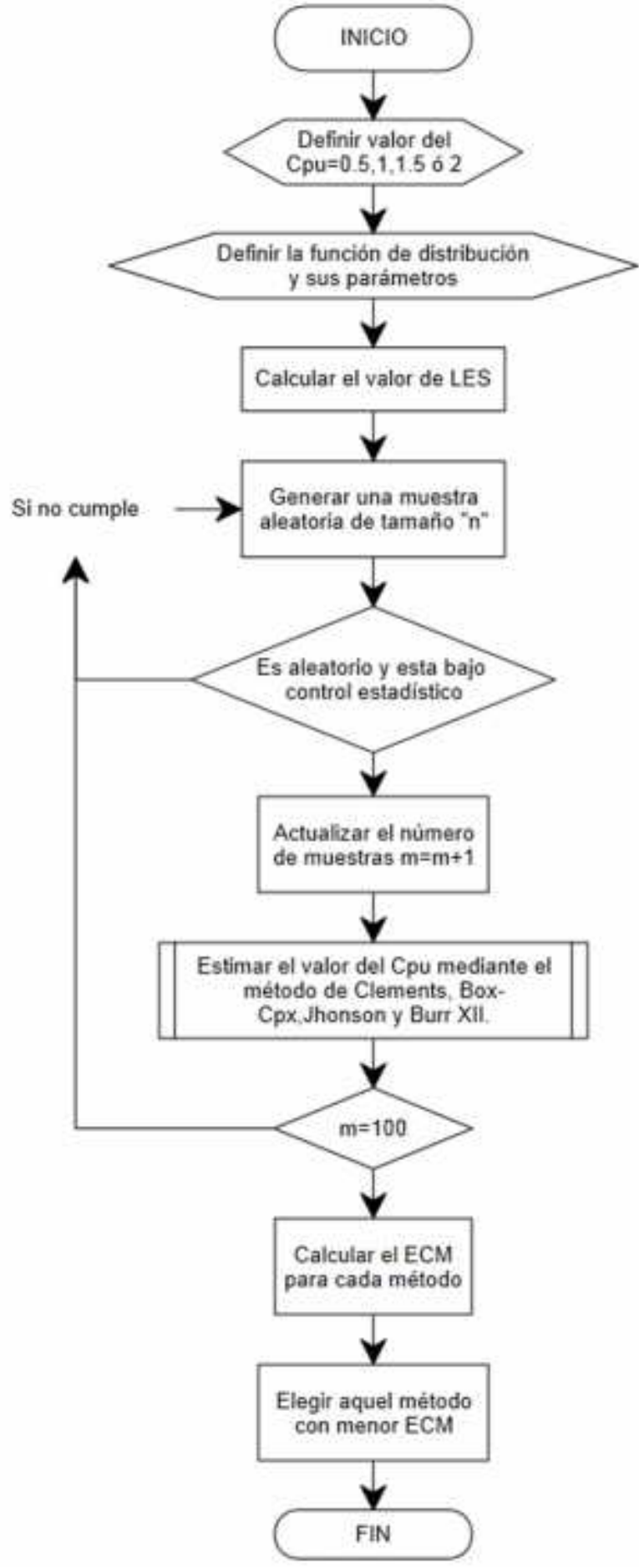

Fig. 4 Proceso del estudio de simulación.

\section{RESULTADOS}

En esta sección se presenta el esquema de los resultados obtenidos, según el proceso de simulación, para los niveles elegidos del $\mathrm{Cpu}$, de la distribución y de los niveles de asimetría. 
Inicialmente se muestra resultados parciales para mostrar como fue el comportamiento de las estimaciones según el proceso de simulación.
En la Fig. 5 se muestra el resultado para $C p u=1$ con distribuciones Beta(5,3), Gamma(27,1), Weibull $(5,1.5)$ y Lognormal $(1,0.75)$.
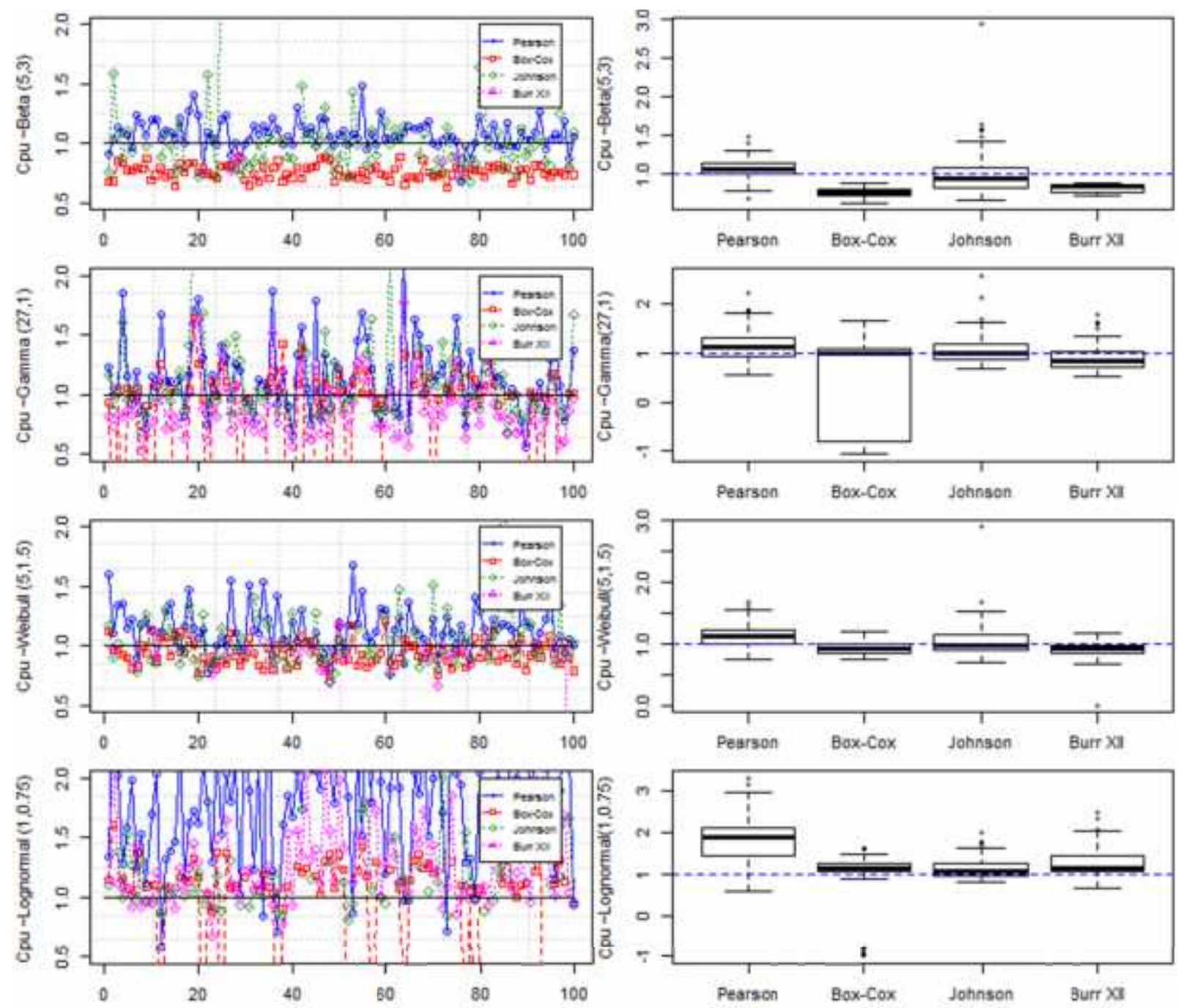

Fig. 5 Resultados para Beta(5,3), Gammi $(27,1)$, Weibull(5, 1.5) y Lognormal $(1,0.75), C p u=1$.

Según la Fig. 5 se puede observar que; primero, para la distribución Beta $(5,3)$, el método de Percentiles con el ajuste de distribución de Pearson presenta mejores resultados ya que las estimaciones están más cercanas a $\boldsymbol{C p} \boldsymbol{u}=\mathbf{1}$ y presentan menor variabilidad. Segundo, para la distribución Gamma(27,1), el método de Percentiles con el ajuste de distribución de Bur XII obtiene valores más cercanos a $\boldsymbol{C p} \boldsymbol{u}=\mathbf{1}$ con menor variabilidad, sin embargo los demás métodos también presentan buenos resultados pero con mayor variabilidad o sesgo. Tercero, en el caso de la distribución Weibull $(5,1.5)$, aunque los cuatro métodos presentan resultados satisfactorios para la estimación de $\mathbf{C} \boldsymbol{p} \boldsymbol{u}=\mathbf{1}$, ê método de transformación de Box-Cox es el mejor. Finalmente, para la distribución Lognormal(1,0.75), el método de transformación de Johnson es el más adecuado. 
Como se mencionó anteriormente, en la Fig. 5, solo se muestra una parte de los resultados del estudio de simulación con fines de comprobar y confirmar que cada uno de los métodos presentados es mejor en ciertas situaciones y por lo tanto se niega el paradigma de que alguno de los métodos presentados sea el mejor y el más adecuado en cualquier situación cuando se trabaja con datos no normales. En la Fig. 6 y Fig. 7 se muestra el resultado general del estudio de simulación para $C p u=0.5,1,1.5$ y 2 y para las distribuciones mencionadas en la metodología de simulación y comparación según el ECM (Error Cuadrático Medio) obtenido por cada método. En estas figuras los mejores métodos, aquellos con menor ECM están representados de color rojo. Además, Los resultados "NA" significan que el método no pudo realizar alguna estimación en las 100 muestras simuladas. Los resultados de la Fig. 5 pueden corroborarse, según el ECM obtenido, en la Fig. 6 y Fig. 7.



Fig. 6 Resultados para Cpu $=0.5$ y Cpu $=1$ segin ECM.

\begin{tabular}{|c|c|c|c|c|c|c|c|c|c|c|c|c|c|c|}
\hline \multirow[b]{3}{*}{ Distrithución } & \multirow[b]{3}{*}{ Parimetrul } & \multirow[b]{3}{*}{ Darimetroz } & \multirow[b]{3}{*}{$\begin{array}{c}\text { Aplametria } \\
\text { (Seigo) }\end{array}$} & \multirow[b]{3}{*}{$\begin{array}{l}\text { Categoria de } \\
\text { ta Asimetria }\end{array}$} & & & & & & & & & & \\
\hline & & & & & \multicolumn{4}{|c|}{ Cpu-1.5 } & \multicolumn{6}{|c|}{ Cpu=2 } \\
\hline & & & & & Fearson & Bescos & Jehnsen & Burr XII & Paaraen & & Son-Cen & Johnson & & Burr XII \\
\hline \multirow{5}{*}{ Beta } & formales & forma2 21 & -1.47 & Niegative alto & 20.0055 & 술 0.5453 & (5) 0.1753 & NA & \& 0.0263 & tî & 0.5604 & 240.2565 & NA & \\
\hline & tormalas & forma 223 & 0.1 & Negrtive moderado & 20.047 & (11.143 & 울 0.2144 & St 0.0953 & 80.0051 & 8 & 0.1750 & 욤 0.5905 & 8 & 0.1126 \\
\hline & forme $1=6$ & forma $2=6$ & 0. & Simetrice & B. 0.0708 & B a a 0535 & 술 0.2533 & 18.00613 & 8.1259 & 8 & 0.1154 & 0.6452 & 18. & 0.1105 \\
\hline & formalas & focma $2=5$ & 0.3 & Positive maderado & 3. 0.0952 & 0.1080 & 살 0.2666 & \& 0.1158 & 80.1723 & $\Rightarrow$ & $0.25: 2$ & 0.3687 & 13. & 0.2082 \\
\hline & formala 1 & forma $2=8$ & 1.42 & Fositive alte & 3.0 .0040 & 0.1799 & 0.2231 & $\Rightarrow 0.1590$ & 2. 0.1494 & 2 & 0.5435 & 0.7024 & N & 0.2855 \\
\hline \multirow{2}{*}{ Gamma } & forman 27 & ercalinn 1 & 0,34 & Focitivo moderado & 80.2560 & 2.0038 & 8.1676 & 18. $0.16+0$ & 0.4569 & f合 & 3.2622 & 0.3637 & 8 & 0.2937 \\
\hline & formax 2 & escalary 15 & 241 & Positivo alto & to. 0.4393 & B. 0.0734 & D.1919 & 1.0.0791 & 울 0.7772 & $y$ & 0.2828 & $\Rightarrow 0.4424$ & 8 & 0.1396 \\
\hline \multirow{4}{*}{ Weibult } & forma=5 & escaile $=1.5$ & 0.28 & Negstivo mederado & 270.1164 & (1) 00400 & 0.1634 & $\Rightarrow 0.1065$ & $\Rightarrow 0.2098$ & 8 & 0.0997 & 0.3085 & $\Rightarrow$ & 0.1917 \\
\hline & forman3.5 & escala=1.5 & 0 & Simêtrice & Q10.1107 & 20.0436 & 0.2585 & 8. 00364 & 0.1978 & t5 & 2.1010 & 0.4372 & If & 0.0653 \\
\hline & formana.5 & ascalan3.5 & 0.4 & Positive moderado & $\Rightarrow 0.1640$ & 18.0.0504 & 삼 0.2532 & B. 00432 & $\Rightarrow 0.2967$ & 8 & 0.1364 & 0,4714 & 8 & 0,0393 \\
\hline & forman 1 & escala $=0.5$ & 197 & Positivo alte & 10.7338 & 80.1154 & 80.0961 & 3.00784 & te 1.3048 & Q & 0.4148 & 0.4469 & 16 & 0.1393 \\
\hline \multirow{2}{*}{ tognormal } & mesuleg:-10. & stllog $=0.25$ & 0.30 & Posituso moderado & 8.03873 & 술 2.3900 & 0.2008 & 8. 0.1400 & 1) 0.6945 & 살 & 3.7979 & (8. 0.3470 & 8 & 02538 \\
\hline & meanloge1 & stlog=0.75 & 3.26 & Postitivoalte & ․․ㄹ 2.2973 & 0.3836 & 1. 0.1550 & B. 0.4267 & 10.0624 & $\Delta$ & 1.4650 & 18. 0.4443 & 8 & 0.7618 \\
\hline
\end{tabular}

Fig. 7 Resultados para $C p u=1.5$ y Cpu $=2$ seguin ECM.

\section{CONCLUSIONES}

Los resultados obtenidos dan muestra de que ninguno de los métodos es el mejor estadísticamente en cualquier situación de no normalidad de los datos. Por ello el método más adecuado depende del tipo de distribución, del nivel de asimetría y del valor aproximado del ICP. 
Además, los resultados presentados son útiles para identificar el escenario bajo el cual se trabaja y determinar que método emplear con fines de asegurar que se obtendrá resultados más confiables al resto de los métodos presentados.

En el proceso de simulación existen complicaciones para estimar los parámetros de la distribución de Burr XII, así que se recomiendo emplear algoritmos eficientes para este proceso.

Finalmente, se deja claro que aún está abierto el campo de investigación de los ICP con métodos bayesianos, multivariados, computacionales entre otros.

\section{BIBLIOGRAFÍA}

1. Juran, J. M., "Quality Control Handbook2", 3rd ed. New York:, 1974, pp. 1600.

2. Kane, V. E., "Process Capability Indices", Journal of Quality Technology, vol. 2097, no. 7, pp. 41-52, 1986.

3. Chan, L. K., Cheng, S. W., Spiring, F. A., "A new measure of process capability: Cpm", Journal of Quality Technology, vol. 20, no. 3, pp. 162-175, 1988.

4. Pearn, W. L., Kotz, S., Johnson, N., L., "Distributional and inferential properties process capability indices", Journal of Quality Technology, vol. 24, no. 4, pp. 216-233, 1992.

5. Kotz, S., Johnson, N. L.,"Process Capability Indices", 1st ed. Chapman and Hall/CRC, 1993, p. 224.

6. Box, G. E. P., Cox, D. R., "An Analysis of Transformations", Journal of Royal Statistical Society, vol. B, no. 26, pp. 211-252, 1964.

7. Chou, Y. M., Polansky, A. M., Mason, R. L., "Transforming non-normal data to normality in statistical process control", Journal of Quality Technology, vol. 30, 1998.

8. Clements, J. A., "Process capability calculations for non-normal distributions", Quality Progress, no. 22, pp. 95-100, 1989.

9. Liu P. H., Chen, F.-L., "Process capability analysis of non-normal process data using the
Burr XII distribution", The International Journal of Advanced Manufacturing Technology, vol. 27, no. 9-10, pp. 975-984, Aug. 2005.

10. Ahmad, S., Abdollahian, M., "Process capability estimation for non-normal quality charactersitics using Clement, Burr and Box-Cox methods", ANZIAM, vol. 49, pp. 642665, 2008.

11. Hosseinifard, S. Z., Abbasi, B., Ahmad, S., Abdollahian, M., "A transformation technique to estimate the process capability index for non-normal processes", The International Journal of Advanced Manufacturing Technology, vol. 40, no. 5-6, pp. 512-517, Feb. 2008.

12. Czarski, A., "Estimation of process capability indices in case of distribution unlike the normal one", Archives of Materials Science, vol. 34, no. 1, pp. 39-42, 2008.

13. Chen, J., Ding, C., "A new process capability index for non-normal distributions", International Journal of Quality, vol. 18, no. 7, pp. 762-770, 2001.

14. Pearson, K., "Contributions to the Mathematical Theory of Evolution. II. Skew Variation in Homogeneous Material", Philosophical Transactions of the Royal Society A: Mathematical, Physical and Engineering Sciences, vol. 186, pp. 343-414, Jan. 1895.

15. Elderton, W., "Systems of frequency curves", Chemistry \&amp; 1969.

16. Burr, I., "Cumulative frequency functions", Annals of Mathematical Statistics, vol. 13, no. 2, pp. 215-232, 1942.

17. Fermín, J., Valdiviezo, M., "Estimación de índices de capacidad de procesos usando la distribución generalizada de pareto", pp. 93106, 2010.

18. Takahasi, K., "Note on the Multivariate Burr's Distribution", Annals of the Institute of Statistical Mathematics, vol. 17, no. 1, pp. 257-260, 1965.

19. Kleiber, C., "A Guide to the Dagum Distributions", December, 2007.

Correspondencia: jose.camero@gmail.com

Recepción de originales: enero 2014

Aceptación de originales: abril 2014 